\section{Designing PCR Primers on the Web}

\section{Mildad Biñas, eBioinformatics, Sydney, Australia (mildad.binas@ebioinformatics.com)}

PCR is perhaps the single most significant advancement in molecular biology in over a decade. Not only has PCR greatly influenced the amount of sequence information currently available, but it is also changing the face of disease diagnosis, food microbiology and even forensic science.

Among all the parameters employed in PCR, perhaps the most critical is primer design. The length of the amplification product, its melting temperature and the total product yield of a reaction are all influenced by the quality of the primer sequences. Several guidelines can be followed when designing primers (5). There are numerous online tools dedicated to helping molecular biologists design effective PCR primers. Most sites have been designed to allow users to easily modify any parameter that affects the design of effective primers.

\section{Primer3}

Primer3 (9) is one of the most popular primer design tools available online (see below) and is based on programs Primer 0.5 , written by S. Lincoln, M. Daly and E. Lander (6), and Primer v2, written by R. Resnick. For any given sequence, Primer 3 determines which subsequence is most suitable for amplification by first using the information about target region and/or product length as specified by the investigator. Once the target region(s) has been determined, Primer3 then looks for regions that may be significantly similar to repeat elements, if any are specified, and further restricts potential target regions for amplification. The $T_{m}$ for potential primers are then calculated as previously described $(2,10)$. Primers that pass these criteria are paired, and the potential product length for each pair is calculated. The primer pairs' self and pairwise complementarity are assessed, and their sequences are again checked for similarity to repeat sequences using a slower, more sensitive method than what was initially used. The program then chooses the optimal primers from the list generated; one pair of primers is suggested for each range of product length requested.

Primer3 is available at the Center for Genome Research at the Whitehead Institute for Biomedical Research (http://www.genome.wi.mit.edu/cgi-bin/primer/ primer3_www.cgi), where an older Web implementation of the same program (http://www.genome.wi.mit.edu/ cgi-bin/primer/primer3.cgi) and the Primer3 source code are also available. Local copies of this older implementation are located at several other sites, including the University of Cambridge's Department of Pathology (http://www.path.cam.ac.uk/cgi-bin/primer3.cgi), Northwestern University Medical School (http://www. basic.nwu.edu/biotools/Primer3.html), Marquette University Biomathematics Computational laboratory
(http://eve.mscs.mu.edu/RESEARCH/PPICK/pp_info. html) and at the Department of Biological Services at Weizmann Institute of Science, Israel (http:// bioinformatics.weizmann.ac.il/cgi-bin/primer/primer3. cgi), while a beta version of the latest implementation is found at the Oxford University Bioinformatics Centre (http://www.molbiol.ox.ac.uk/cgi-bin/primer3_www. cgi). These sites allow users to cut and paste a single nucleotide sequence into a form and set all the various parameters including melting temperature, size, GC content, PCR product size, positional restrictions within the source sequence and other miscellaneous restrictions such as sequence quality information. These sites also allow investigators to change the default penalty weights of each criterion that Primer3 uses to find the most optimal primers. The latest Web implementations found at the Whitehead Institute for Biomedical Research and the Oxford University Bioinformatics Centre also allow investigators to enter sequences of potential primers and test them on the specified criteria.

Primer3 is also available through the Web-based sequence analysis service provider, BioNavigator ${ }^{\circledR}$ (http://www.bionavigator.com) as one of several PCR primer design tools. Sequences can either be cut and pasted, uploaded from the investigator's computer in a variety of known file formats or obtained directly from one of the databases maintained onsite. The output generated by Primer3 at this site allows investigators to select the sequences and save them as query sequences to BLAST and other database similarity searching programs. Moreover, investigators can use multiple sequences to design primers by using tools that extract the consensus sequence from a multiple sequence alignment.

\section{Prime}

Prime, which is distributed as part of the Wisconsin Package from the Genetics Computer Group, is also a wellknown program for primer design. This program calculates primer melting temperatures using the nearest neighbor model (1) calculation determined by Santalucia (11), while product melting temperatures are determined using the formula as modified by Rychlik et al. (10). Like Primer3, this program also checks primer self and pairwise complementarity as described by Hillier and Green (4). Prime allows investigators to set values for the standard design parameters, which include primer and product length, primer GC content, primer melting and annealing temperatures. This program also allows investigators to test potential primer sequences.

Prime is found in many bioinformatics Web servers, including BioNavigator, the Human Genome Mapping Project, UK (http://www.hgmp.mrc.ac.uk/), the Danish Biotechnological Database, BioBase, at the University of Aarhus, Denmark (http://biobase.dk/index.html) and the Biotechnology Computing Facility at the University of Arizona (http://bcf.arl.arizona.edu/gcg.html).

\section{CODEHOP}

CODEHOP is a useful degenerate primer design tool for 
Quebecor - On-Ramp graphic goes here run visible portion of black circle in red - left align with text

designing primers to amplify genes encoding members of specific protein families, starting from the sequence of other protein family members. It produces a set of primers with each primer consisting of a short degenerate $3^{\prime}$ core region and a longer $5^{\prime}$ consensus clamp region (8). CODEHOP accepts a set of blocks as input, where a block is an aligned array of amino acid sequence segments without gaps representing a highly conserved region of homologous proteins (7). A position-specific scoring matrix is then computed for each block using the odds-ratio method, which computes weights on diversity observed at each alignment position, rather than traditional weighting method assumptions (3). One consensus residue is selected for each position in the block, and the most common codon corresponding to each consensus residue is selected using the codon usage table specified by the investigator. The DNA position scoring matrix, which is calculated using the position-specific amino acid matrix, is then used to determine the most likely codon for each DNA position, and the high-scoring nucleotide residues are considered for the consensus clamp regions. Possible degenerate regions are identified by scanning the DNA matrix generated in the $3^{\prime} \rightarrow 5^{\prime}$ direction, looking for an invariant $3^{\prime}$ nucleotide position, a length of 11-12 positions ending on a codon boundary and have a maximum degeneracy, the product of the number of possible bases at each position, of 128 . These candidate degenerate regions are then extended using the $5^{\prime}$ consensus clamp regions previously identified; the length is controlled by $T_{m}$ calculations (8).

CODEHOP is available at the Fred Hutchinson Cancer Research Center (http://www.blocks.fhcrc.org/), its mirror site at the Weizmann Institute of Science (http:// bioinformatics.weizmann.ac.il/blocks/index.html) and at BioNavigator. Programs for generating the sequence blocks required as input by CODEHOP are also available at the same sites.

\section{Other Online Primer Design Sites}

There are several other sites dedicated to primer design. GeneFisher is a primer design tool (http://bibiserv. techfak.uni-bielefeld.de/genefisher/) that allows investigators to use as input either single sequences or multiple closely related ones. Multiple sequences can be aligned before using this tool, but if sequences are entered unaligned, they are first aligned using ClustalW (13) or DCA (Divide-and-Conquer Multiple Sequence Alignment), a program for producing fast, high-quality multiple sequence alignments using the DCA algorithm, a heuristic approach to sum-of-pairs optimal alignment (12). GeneFisher is intended for those unfamiliar to primer design because it guides the users through each step. The Primer Generator (14) is particularly useful for designing primers appropriate for site-directed mutagenesis experiments. It analyzes the nucleotide sequence and its corresponding amino acid sequence and designs a primer that either has a new restriction enzyme site or is missing an old one, which helps in differentiating between mutated and nonmutated sequences. Primer Generator is located at http://www. med.jhu.edu/medcenter/primer/primer.cgi. Other online primer design tools include the Virtual Genome Center's Web Primers, (http://alces.med.umn.edu/webprimers. html), Primers! for the World Wide Web at Williamstone Enterprises, (http://www.williamstone.com/), DoPrimer at the Virtual Laboratory at Interactiva (http://doprimer. interactiva.de/), Stanford University's Web Primer (http://genome-www2.stanford.edu/cgi-bin/SGD/webprimer/) and Primer Selection at the Baylor College of Medicine Search Launcher (http://dot.imgen.bcm.tmc. edu:9331/seq-util/seq-util.html).

The large number of programs for PCR primer design reflects the central role of PCR in modern molecular biology. It is important to remember that all these programs are designed to assist in the primer design process and are not meant to replace the eye of the experienced researcher, especially considering the sometimes unpredictable nature of PCR experiments. When planning important PCR experiments, it is usually worthwhile to compare the predictions of several different programs and to use common sense and laboratory experience to evaluate the suggested primers before committing to their synthesis.

\section{REFERENCES}

1.Borer, P.N., B. Dengler, I. Tinoco, Jr. and O.C. Uhlenbeck. 1974. Stability of ribonucleic acid double-stranded helices. J. Mol. Biol. 86:843853.

2.Breslauer, K.J., R. Frank, H. Blocker and L.A. Marky. 1986. Predicting DNA duplex stability from the base sequence. Proc. Natl. Acad. Sci. USA 83:3746-3750.

3.Henikoff, J.G. and S. Henikoff. 1996. Using substitution probabilities to improve position-specific scoring matrices. CABIOS 12:135-143.

4.Hillier, L. and P. Green. 1991. OSP: a computer program for choosing PCR and DNA sequencing primers. PCR Methods Appl. 1:124-128.

5.Innis, M.A. and D.H. Gelfand. 1994. Optimization of PCRs, p. 13-20. In M.A. Innis, D.H. Gelfand, J.J. Sninsky and J.J. White (Eds.), PCR Protocols: A Guide to Methods and Applications. CRC Press, London.

6.Lincoln, S.E., M.J. Daly and E.S. Lander. 1991. PRIMER: a computer program for automatically selecting PCR Primers (http://www.genome. wi.mit.edu/ftp/distribution/software/primer.0.5/).

7.Posfai, J., A.S. Bhagwat, G. Posfai and R.J. Roberts. 1989. Predictive motifs derived from cytosine methyltransferases. Nucleic Acids Res. 17:2421-2435

8.Rose, T.M., E.R. Schultz, J.G. Henikoff, S. Pietrokovski, C.M. McCallum and S. Henikoff. 1998. Protein consensus-degenerate hybrid oligonucleotide primers for amplification of distantly related sequences. Nucleic Acids Res. 26:1628-1635.

9.Rozen, S. and H.J. Skaletsky. 1996-1998. Primer3. Code available at http://www.genome.wi.mit.edu/genome_software/other/primer3.html.

10.Rychlik, W., W.J. Spencer and R.E. Rhoads. 1990. Optimization of the annealing temperature for DNA amplification in vitro. Nucleic Acids Res. 18:6409-6412.

11.Santalucia, J., Jr. 1998. A unified view of polymer, dumbbell, and oligonucleotide DNA nearest-neighbor thermodynamics. Proc. Natl. Acad. Sci. USA 95:1460-1465.

12.Stoye, J., V. Moulton and A.W. Dress. 1997. DCA: an efficient implementation of the divide-and-conquer approach to simultaneous multiple sequence alignment. CABIOS 13:625-626,

13.Thompson, J.D., D.G. Higgins and T.J. Gibson. 1994. CLUSTAL W: improving the sensitivity of progressive multiple sequence alignment through sequence weighting, position-specific gap penalties and weight matrix choice. Nucleic Acids Res. 22:4673-4680.

14. Turchin, A. and J.F. Lawler, Jr. 1999. The primer generator: a program that facilitates the selection of oligonucleotides for site-directed mutagenesis. BioTechniques. 26:672-676. 